\title{
Habilidades Visoperceptivas y Perfil Psicomotor: Análisis de una Muestra de Estudiantes en Etapa Preescolar
}

\author{
Visuoperceptive Skills and Psychomotor Profile: Analysis of a \\ Sample of Preschool Students
}

Iris Sol Ovalle Cijanes ${ }^{1 *}$ (i)

Recibido: 07-09-2020; Aceptado: 07-12-2020; Publicado: 20-12-2020

\section{RESUMEN}

El objetivo de esta investigación fue analizar la relación entre la percepción visual y la psicomotricidad en niños en edad preescolar en la ciudad de Cúcuta. Método. se llevó a cabo mediante metodología cuantitativa no experimental, con alcance descriptivo y correlacional de corte transversal, con 30 participantes, mediante muestreo intencionado de acuerdo con los intereses de la investigadora. Resultados. Se encontró que el desempeño más bajo se dio en la percepción visual con respuesta motriz reducida (PMR). Se encontró que la tonicidad y la lateralidad presentan las puntuaciones más altas en la muestra. Conclusiones. La percepción visual y la psicomotricidad poseen una relación directa en los estudiantes de educación preescolar para este estudio.

Palabras clave: Percepción Visual; Psicomotricidad; Sensorial; Cognición.

\begin{abstract}
The objective of this research was to analyze the relationship between visual perception and psychomotor skills in preschool children in the city of Cúcuta. Method. it was carried out using a non-experimental quantitative methodology, with a descriptive and correlational cross-sectional scope, with 30 participants, through intentional sampling according to the researcher's interests. Results. It was found that the lowest performance was in visual perception with reduced motor response (PMR). Tonicity and laterality were found to have the highest scores in the sample. Conclusions. Visual perception and motor skills have a direct relationship in preschool students for this study.
\end{abstract}

Keywords: Visual Perception; Psychomotor; Sensory; Cognition.

${ }^{1}$ Institución Educativa San Pedro Calver, Colombia, irisovalle.cijanes@gmail.com 


\section{INTRODUCCIÓN}

El desarrollo de la lectoescritura fundamenta las bases educativas y sociales del individuo, el cual implica que se fomenten los procesos psicológicos básicos, como la memoria, la percepción, la conciencia y los procesos cognitivos y metacognitivos entre otros (López-Olóriz et al., 2020). Es así que se consolida el desarrollo de adquisición de las diferentes funciones neuropsicológicas de tipo cortical y subcortical que facilitan la adquisición de herramientas que determinan el aprendizaje de la lectura y escritura del lenguaje (Mariángel \& Jiménez, 2015).

Como menciona González et al. (2015), los procesos de lectoescritura fundamentan el aprendizaje, influyen en la transmisión cultural de conocimientos y a su vez determinan el establecimiento de las competencias básicas que facilitan el fomento del aprendizaje. De esta manera, RamírezLeyva (2016) afirma que la lectura es la herramienta universal mediante la cual se adquiere información, se realizan procesamientos cognitivos y se afianzan las estrategias de comprensión que conllevan al individuo al desarrollo de capacidades, habilidades y conocimientos.

Es así que el desarrollo de las destrezas motrices presentan los indicativos asociados a la maduración cerebral; de esta manera, la psicomotricidad a través del movimiento es el indicador de los elementos básicos dentro del proceso del aprendizaje (Gutiérrez \& Castillo, 2014). La consecución de movimientos bimanuales de forma coordinada representa la precisión motora, así como la adecuada organización espacial que facilita el desarrollo de las destrezas en la relación existente entre lectura y escritura, propiciando los primeros aprendizajes que incluyen la integración del área motriz y la actividad psíquica que se ha conceptualizado como psicomotricidad (Campo, 2011).

El ojo es el órgano del aparato visual, capaz de abstraer la información del exterior por medio de imágenes que atraviesan las diferentes capas del glóbulo ocular, concentrándose en la retina que abstrae la información del mundo exterior. Como indica Cabrales (2015), la clasificación realizada por Brodmann, divide las áreas 17, 18 y 19 que componen el lóbulo occipital; el área 17 corresponde al córtex visual primario; en el área 18 se ubica la región paraestriada, su función principal es la elaboración y a su vez síntesis del contenido de la información visual; a su vez el área 19 proporciona la integración multimodal de todas las informaciones visuales que, al unirse con las áreas posteriores del lóbulo parietal, forma el sitial de la memoria visual (SánchezPanchuelo et al., 2014).

Siguiendo a Alesi et al. (2019), existen tres tipos de sensaciones, las exteroceptivas, cuya función se centra en proporcionar la información que se encuentra en el entorno, por su parte las sensaciones propioceptivas encargadas de las sensaciones que se encargan de la comunicación del cuerpo de forma cinestésica y vestibular y por su parte las sensaciones interoceptivas presentan la información que ocurre al interior del cuerpo como lo es el calor, el frio, la sed y el hambre entre otros.

La psicomotricidad desde el modelo de Luria, plantea el funcionamiento del sistema nervioso a través del movimiento que facilita el desarrollo global de las áreas afectivas, social, cognitiva y motora, donde se ponen de manifiesto los procesos que concretan la motricidad y el lenguaje humano (Bora et al., 2019).

Por lo anterior, la percepción visual y la psicomotricidad hacen parte del desarrollo neuropsicológico de los infantes. Como lo menciona Torres-Rivas \& López-Gómez (2017), la planificación y la ejecución de los movimientos motores, precisa de la adquisición de la información visual para potenciar las habilidades de percepción que puntualizan los movimientos. Es así que el grado de desarrollo motriz refleja el nivel de maduración cerebral que es adquirido; de esta manera, se configura una organización espacial y motora (Gago- Galvagno \& Elgier, 2018). 
Ahora bien, desde el enfoque cognitivo, los aportes de (Rodriguez \& Castillo, 2018) afirman la relación entre la percepción visual y la asociación desde lo psicológico y lo biológico, que parte desde el proceso sensorial y perceptual y permite abarcar el proceso mental que se encarga de interpretar y organizar las sensaciones con lo cual se le asigna un significado. De la misma forma, Pascual-Lacal et al.(2018) indican que la representación de la información externa permite la adquisición de conocimientos en los infantes, de tal manera que pueden descubrir los objetos, sus cualidades, texturas, colores, tamaños, olores y, a su vez, sabores que conforma la red de instrucciones adquiridas y les permite la formación de los procesamientos cognitivos superiores, como lo son la inteligencia y el pensamiento.

\section{MÉTODO}

Diseño. El proceso investigativo se realizó desde el enfoque cuantitativo no experimental con alcance descriptivo y correlacional de corte transversal (Hernández et al., 2014), donde se relacionó las variables del nivel de percepción visual y el perfil psicomotor de un grupo de estudiantes en edad preescolar.

Participantes. Esta investigación contó con una $n=30$ participantes de un jardín infantil en la ciudad de Cúcuta, cuyos participantes tienen rangos de edad de 4 y 6 años, 17 niños y 13 niñas que pertenecen al grado de educación preescolar. Quienes realizaron su proceso de participación, mediante el proceso de consentimiento informado por parte de sus padres y en colaboración con la institución educativa, siguiendo los lineamientos de la Ley 1090 de 2006 (Congreso de la República de Colombia, 2006) que sustenta el ejercicio bioético e investigativo en Colombia y en consonancia con la declaración de Helsinki (Asociación Médica Mundial, 2001) y la Resolución 008430 del (Ministerio de Salud y Protección , 2012), este estudio se clasificó como investigación de riesgo mínimo.

Instrumentos. Se utilizó el Método de Evaluación de la Percepción Visual de Frostig, DTVP - 2 (Hammill et al., 1995), compuesto por ocho subpruebas que miden la coordinación ojomano, posición en el espacio, copia, figura-fondo, relaciones espaciales, cierre visual, velocidad visomotora y constancia de forma, las cuales miden la habilidad perceptiva visual. Las subpruebas realizan una clasificación de respuestas motrices reducidas y realzadas lo cual permite que se obtenga un cociente de percepción visual general (PVG) y un cociente de Integración Visomotora (IVM), realizando la medición de las variaciones presentadas en el rendimiento de cada habilidad evaluada.

Por otra parte, se utilizó la batería psicomotora- BPM (Da Fonseca, 2005), que permite identificar los déficits funcionales que se presentan a nivel motriz. Se encuentra dividida en siete factores: tonicidad, equilibrio, lateralidad, noción de cuerpo, estructuración espacio temporal, praxia global y praxia fina. A su vez se dividen en subfactores que permiten identificar el perfil psicomotor de los participantes.

Procedimiento. Se realizó una reunión que incluyó a las directivas, profesores y padres de familia de la institución educativa, con el objeto se socializar el objetivo de la investigación, así como los instrumentos a utilizar. En el caso de los padres que de manera voluntaria autorizaron la participación de los niños en la investigación, se procedió a diligenciar el consentimiento informado: una vez se realizó la firma del documento, se procedió a codificar la información con fines de salvaguardar la confidencialidad de los participantes y sus padres (Cortés \& Iglesias, 2004).

Los estudiantes fueron evaluados de forma individual, con una duración aproximada de 1 h 30 min en las instalaciones de la institución educativa. El proceso evaluativo fue realizado por tres semanas consecutivas y cada día fueron evaluados dos niños dentro de la jornada escolar. 


\section{RESULTADOS}

Con el fin de realizar una descripción de los resultados obtenidos, en primer lugar se presentan los estadísticos descriptivos, que consistieron en los cálculos de las medidas de tendencia central y la desviación típica calculada por las subpruebas (Tabla 1).

Tabla 1. Medidas de dispersión de las habilidades visoperceptivas

\begin{tabular}{ccc}
\hline & Media & Desviación típica \\
\hline OM & 10,60 & 2,66 \\
PE & 10,27 & 2,19 \\
CO & 10,13 & 1,87 \\
FF & 8,10 & 2,36 \\
RE & 9,93 & 2,11 \\
CV & 7,97 & 1,73 \\
VVM & 11,47 & 2,90 \\
CF & 11,43 & 1,67 \\
PVG & 100,27 & 9,77 \\
PMR & 94,77 & 11,10 \\
IVM & 103,53 & 10,58 \\
N = 30 & & \\
\hline
\end{tabular}

OM (coordinación ojo-mano), PE (posición en el espacio), CO (copia), FF (figurafondo), RE (relaciones espaciales), CV (cierre visual), VVM (velocidad visomotora) y CF (constancia de forma) PVG (percepción visual general) PMR (percepción visual con respuesta motriz reducida) e IVM (Integración visomotora).

Respecto a lo descrito, se identifica que en la muestra las habilidades de cierre visual figura- fondo y relaciones espaciales obtuvieron bajas puntuaciones, indicando de esta manera dificultades en el reconocimiento y discriminación de figuras ocultas dentro de un grupo de imágenes y, a su vez, dificultades en los procesos de abstracción de representación del mundo a través de simbolismos (Bravo- Cóppola, 2014) la relevancia de conocer el nivel de desempeño de los y las niñas en el uso de las destrezas perceptuales; nivel de desempeño que incide directamente en el desarrollo de competencias en el ámbito académico. Para ello se presenta una Guía de Exploración Diagnóstica que consiste en la presentación de una definición conceptual y operacional de cada una de las sub-áreas de la Percepción Visual y Auditiva (las áreas más vinculadas con el aprendizaje de la lectura y la escritura. A su vez, los cocientes que integran el PMR (percepción visual con respuesta motriz reducida) alcanzaron la puntuación más baja. lo que permite identificar en la muestra mayor grado de dificultades en la integración visomotora (Tabla 2).

Tabla 2. Medidas de dispersión de los factores y perfil psicomotor.

\begin{tabular}{cccc}
\hline & N & Media & Desviación típica \\
\hline F. TON & 30 & 2,67 & 0,47 \\
F. EQUI & 30 & 2,50 & 0,57 \\
F. LAT & 30 & 3,27 & 0,45 \\
F. NOC CU & 30 & 2,43 & 0,56 \\
F. ESP-TEMP & 30 & 1,47 & 0,50 \\
F. PRA GLOB & 30 & 2,40 & 0,62 \\
F. PRA FIN & 30 & 1,93 & 0,69 \\
PERFIL PSM & 30 & 16,60 & 2,22 \\
$\mathbf{N}$ & 30 & & \\
\hline
\end{tabular}

Tonicidad (F. TON), Equilibrio (F. EQUI), Lateralidad (F. LAT), Noción del cuerpo (F. NOC CU), Estructuración espaciotemporal (F. ESP-TEMP), Praxia global (PRA GLOB) y Praxia fina (PRA FIN) 
En los resultados obtenidos en la representación de los factores psicomotores utilizados en la medición - por medio del BPM Tonicidad (F. TON), Equilibrio (F. EQUI), Lateralidad (F. LAT), Noción del cuerpo (F. NOC CU), Estructuración espaciotemporal (F. ESP-TEMP), Praxia global (PRA GLOB) y Praxia fina (PRA FIN) (Salamanca et al., 2013), se encontró que la tonicidad y la lateralidad presentan las puntuaciones más altas en la muestra, lo que permite identificar mayores sensaciones propioceptivas con una fortaleza del control del funcionamiento del cuerpo. Asimismo, la lateralidad como eje motor independiente presenta un proceso dinámico consecuente del desarrollo cortical (Hernández-Flórez et al., 2016).

Respecto de la estructuración espacio temporal y la praxia global que obtuvieron las puntuaciones bajas, se identificó que los niños presentan dificultades en la organización de las diversas actividades dispuestas, así como en los procesos de programación, regulación y verificación (Palacio-Duran et al., 2017). Por ende, las actividades motoras de tipo secuencial requieren mayor intervención en el fomento de actividades que estimulen las estructuras subcorticales, que hacen parte de los sistemas extrapiramidales.

Respecto a los estudios de tipo correlacional entre las variables descritas, se realizó el análisis por medio del coeficiente de Pearson, con el objeto de medir la dependencia lineal del grado de concordancia de las posiciones relativas establecidas con puntuaciones tipificadas (Tabla 3).

Tabla 3. Correlaciones habilidades visoespaciales y perfil psicomotor.

\begin{tabular}{cccc}
\hline & PVG & PMR & IVM \\
\hline F. TON & $0,542 * *$ & $0,386 *$ & 0,559 \\
& 0,002 Sig. & 0,035 Sig. & 0,001 Sig. \\
F. EQUI & $0,419 *$ & $0,442 *$ & 0,359 \\
& 0,021 Sig. & 0,014 Sig. & 0,001 Sig. \\
F. LAT & 0,219 & 0,096 & 0,223 \\
F. NOC CU & 0,246 Sig. & 0,615 & 0,237 \\
F. ESP-TEMP & 0,245 & 0,027 & 0,327 \\
F. PRA GLOB & 0,191 Sig. & 0,885 Sig. & 0,078 Sig. \\
& $0,593 * *$ & 0,454 & 0,523 \\
PRA FIN & 0,001 Sig. & 0,012 Sig. & 0,003 Sig. \\
PERFI PSM & $0,436 *$ & $0,434 *$ & 0,349 \\
& 0,016 Sig. & 0,017 Sig. & 0,059 Sig. \\
& 0,176 & 0,191 & 0,193 \\
\end{tabular}

* Correlación significativa al nivel de 0.05

** Correlación significativa al nivel de 0.01 ;

El cociente de percepción visual se encuentra correlacionado positiva y significativamente con las áreas de tonicidad, equilibrio, estructuración espacio temporal, praxia global y del perfil psicomotor global, lo que permite identificar la relación que se presenta en las variables de percepción visual y el nivel de desarrollo psicomotor, situación que es ampliamente descrita por la literatura científica, como lo menciona Rodríguez \& Gaeta (2018) desde las aportaciones realizadas que afirman las integraciones en función del organismo, desarrollando habilidades y capacidades desde las funciones cognitivas, volitivas y motivacionales en los niños en etapa escolar.

Desde el punto de vista de la integración visomotora, se halló correlaciones en las áreas de equilibrio y estructuración espaciotemporal, lo cual indica el seguimiento de patrones de movimientos que integran la percepción, determinando, de esta forma, los aportes al desarrollo 
de la etapa mental, incrementando las destrezas visomanuales y el equilibrio postural, acorde con su edad (Noguera Machacón et al., 2013).

Por otra parte, la correlación existente entre el perfil psicomotor y la percepción visual, que es integrada por la percepción visual general, percepción visual con respuesta motriz reducida y la integración visomotora, corroboran los resultados hallados, colocando de manifiesto la planificación de las estrategias pedagógicas y didácticas que desarrollan la percepción visual en los estudiantes como una fortaleza identificada en la muestra (Ardila \& Ostrosky, 2012).

En contraposición no se encontró correlación en las áreas de lateralidad, noción de cuerpo y praxia fina y la percepción visual, con lo cual se describe que las funciones cognitivas implicadas en la percepción deben fortalecerse para desarrollar actividades complejas que permitan el rendimiento académico de los niños, buscando aumentar la capacidad perceptiva de los sujetos, identificadas en las debilidades perceptivas y psicomotoras, lío que conlleva a plantear un plan de intervención en la muestra (Alonso, 2017).

\section{DISCUSIÓN}

En los resultados obtenidos a partir del objetivo propuesto de la identificación de la correlación entre la percepción visual y la psicomotricidad, realizando una valoración por áreas separadas buscando determinar o no la existencia de la correlación, dentro de los hallazgos presentados se encontró que la puntuación de la percepción visual general ( PVG) de los estudiantes que participaron en esta investigación fue de 100.27 puntos, el PVG se encuentra compuesto por la percepción visual con respuesta motriz reducida (PMR) y la Integración Visomotora (IVM) de los cuales se obtuvo un puntaje de 94.77 y 103.53 respectivamente, de estos puntajes se puede deducir que el desempeño más bajo se dio en la PMR, que a su vez se compone de diferentes pruebas como el cierre visual, la constancia de forma, posición en el espacio y figurafondo. Asimismo, en la ejecución de las pruebas de IVM, la cual se encuentra compuesta por coordinación ojo-mano, copia, relaciones espaciales y velocidad visomotora se obtuvo un puntaje superior en comparación con las pruebas PMR (Jadue, 2017).

Cuando se analizan los resultados obtenidos en relación al sexo, se encontró que los niños poseen un nivel superior de percepción visual general, donde el $5.8 \%$ de este grupo se situó por debajo del rango promedio, que comprende entre los 80 - 89 puntos en comparación con las niñas en las cuales se presentó un porcentaje del $30.7 \%$, no obstante, las niñas presentan un rendimiento superior en tareas de percepción visual con respuesta motriz reducida, equiparándolo con los niños los cuales demuestran una mejor integración visomotora, pudiéndose atribuir estos resultados al comportamiento que posee cada género (Ison \& Korzeniowski, 2016).

Por otro lado, observando los resultados de los siete factores psicomotores de los cuales los puntajes más bajos fueron los de estructuración espaciotemporal (1.43), praxia fina (1.93) y praxia global (2.40), distinto de la noción de cuerpo (2.43) y equilibrio (2.50), los cuales presentaron puntajes un poco más elevados, pero que aún se encuentran en el perfil dispráxico; por lo contrario, la tonicidad (2.67) y lateralidad (3.27) obtuvieron los mejores resultados, situándose en un perfil europráxico (Espejo \& Salas, 2004).

En consecuencia, se halló que que la percepción visual y la psicomotricidad en niños de educación preescolar si están relacionadas, demostrando lo anteriormente mencionado por medio de la correlación de Pearson entre las variables de PVG, PMR e IVM y el perfil psicomotor global; del mismo modo quedo demostrado con los tres cocientes de percepción visual y varios factores psicomotores específicos (Mahyolas Pi et al., 2010). 
Se encontró una correlación directa de 0.01 entre la percepción visual y el perfil psicomotor, asimismo la PVG y los factores psicomotores de tonicidad, estructuración espaciotemporal con el mismo puntaje, mientras que el equilibrio y la praxia global de 0.05 , al mismo tiempo PMR muestra una correlación directa y significativa de 0.01 con el perfil psicomotor global del mismo modo se evidencia la relación entre el nivel de Integración Visomotora (IVM) y el perfil psicomotor global.

\section{CONCLUSIONES}

En síntesis, la percepción visual y la psicomotricidad poseen una relación directa en los estudiantes de educación preescolar; del mismo modo, se evidencia que el perfil psicomotor global se sitúa entre el rango normal para la edad; de igual manera el nivel de percepción visual también se encuentra en el promedio, pero al comparar estas evidencias se encuentra un bajo desempeño en las tareas de percepción visual, con respuesta motriz reducida (Torres-Rivas \& López-Gómez, 2017).

Las habilidades en las que se obtuvo un desempeño inferior fueron el cierre visual, la percepción de figura-fondo y las relaciones espaciales, en contraposición con la velocidad visomotora y la constancia de forma donde el rendimiento fue el mejor (Adams, 2013).

Por otra parte, se encuentran los factores psicomotores de los cuales la estructuración espaciotemporal, la praxia fina y la praxia global consiguieron puntuaciones mínimas, en comparación con la lateralidad y la tonicidad en las cuales el rendimiento fue óptimo (Erazo, 2018).

Se diseñó un programa de intervención basándose en los resultados conseguidos, encaminado a optimizar las habilidades visoperceptivas y motrices, con el objeto de contribuir a la población escolar que presenta dificultades a nivel psicomotriz y de la percepción visual.

\section{REFERENCIAS}

Adams A, Simmons $F$, Willis $C$, Porter $S$. The impact of the development of verbal recoding on children's early writing skills. Br J Educ Psychol. 2013;83(Pt 1):76-97. doi:10.1111/j.20448279.2011.02056.x

Alesi, M., Gómez-López, M., \& Bianco, A. (2019). Diferenciación motora y habilidades cognitivas en la edad preescolar. Cuadernos de Psicología del Deporte, 19(1), 50-59.

Alonso, D. (2017). Desarrollo de las habilidades motrices de las personas con discapacidad intelectual a través del proceso cognitivo. Arte y Salud, 19. https://doi.org/10.6035/ Artseduca.2018.19.10

Ardila, A., \& Ostrosky, F. (2012). Guía para el desarrollo neuropsicológico.

Asociación Médica Mundial. (2001). Declaración de Helsinki de la Asociación Médica Mundial. Gaceta Medica de Mexico, 137(4), 387-390.

Bora, L., Thomazini, V., \& De Toni, P. (2019). Assimetria Direita-Esquerda e Desenvolvimento Neuropsicomotor Humano. CES Psicología, 12(1), 54-68. https://doi.org/10.21615/ cesp.12.1.5 
Bravo, L. (2014). Las destrezas perceptuales y los retos en el aprendizaje de la lectura y la escritura. Una guía para la exploración y comprensión de dificultades específicas. Actualidades Investigativas en Educación, 4(1). https://doi.org/10.15517/aie.v4i1.9047

Cabrales, A. (2015). Neuropsicología y la localización de las funciones cerebrales superiores en estudios de resonancia magnética funcional con tareas Neuropsychology and the localization of superior brain functions in fMRI with task studies. Acta Neurológica Colombiana, 31(1), 92-100.

Campo, L. (2011). Características del desarrollo adaptativo en niños de 3 a 7 años de la ciudad de Barranquilla. Psychologia, 5(2), 95-104. https://doi.org/10.21500/19002386.1136

Congreso de la República de Colombia. (2006). Ley 1090 DE 2006. Diario Oficial, 2006(46383), 1-27. http://www.unisabana.edu.co/fileadmin/Documentos/Investigacion/comite_de_etica/ Ley_1090_2006_-_Psicologia.pdf

Cortés, M., \& Iglesias, M. (2004). Generalidades sobre metodología de la investigación. Colección Material Didáctico. http://www.unacar.mx/contenido/gaceta/ediciones/metodologia_investigacion.pdf

Erazo, O. (2018). Dificultades en integración sensorial, afectividad y conducta en estudiantes de una escuela pública. Praxis \& Saber, 9(20), 143-166. https://doi.org/10.19053/22160159. v9.n20.2018.5884

Espejo, L., \& Salas, J. (2004). Correlación entre el Desarrollo Psicomotor y el Rendimiento Escolar, en niños de primer año de Educación Básica , pertenecientes a establecimientos municipales de dos comunas urbanas de la Región Metropolitana. http://revistas.urosario.edu.co/index. $\mathrm{php} / \mathrm{revsalud/article/viewFile/2681/2216}$

Gago, L. G., \& Elgier, Á. M. (2018). Trazando puentes entre las neurociencias y la educación. Aportes, límites y caminos futuros en el campo educativo. Psicogente, 21(40), 476-494. https://doi.org/10.17081/psico.21.40.3087

González, R., Cuetos, F., Vilar, J., \& Uceira, E. (2015). Effects of the intervention on phonological awareness and naming speed on learning to write. Aula Abierta, 43(1), 1-8. https://doi. org/10.1016/j.aula.2014.06.001

Gutiérrez, E., \& Castillo, J. A. (2014). Reflexiones sobre la concepción del cuerpo y del movimiento para una educación integral de la primera infancia. Praxis Pedagógica, 14(15), 15-42. https:// doi.org/10.26620/uniminuto.praxis.14.15.2014.15-42

Hernández-Flórez, C., Beltrán, M., \& Contreras, G. (2016). Análisis de la neurogénesis cortical en el desarrollo cerebral de ratones carentes del receptor de ácido. Universitas Médica.

Hernández, R., Fernández, C., \& Baptista, P. (2014). Metodología de la Investigación. En Mc Graw Hill: Vol. Sexta edic (Números 978-1-4562-2396-0). https://doi.org/10.1017/ CBO9781107415324.004

Ison, M., \& Korzeniowski, C. (2016). El rol de la atención y percepción viso-espacial en el desempeño lector en la mediana infancia. Psykhe, 25(1), 1-13. https://doi.org/10.7764/ psykhe.25.1.761

Jadue, T. (2017). Habilidades visoperceptuales en niños escolarizados de 7 a 12 años con ambliopía refractiva. Ciencia \& Tecnología para la Salud Visual y Ocular, 15(2), 31. https:// doi.org/10.19052/sv.4145 
Ovalle - Habilidades Visoperceptivas y Perfil Psicomotor: Análisis de Estudiantes de Preescolar

López-Olóriz, J., Pina, V., Ballesta, S., Bordoy, S., \& Pérez-Zapata, L. (2020). Petit UBinding project: An efficacy study of a reading acquisition and reading improvement method for first grade children. Revista de Logopedia, Foniatria y Audiologia, 40(1), 12-22. https://doi. org/10.1016/j.rlfa.2019.06.001

Mahyolas, M., Villarroya, A., \& Reverter, J. (2010). Relación entre la lateralidad y los aprendizajes escolares. Apunts. Educación Física y Deportes, 101(3), 32-42. http://repositori.udl.cat/ handle/10459.1/46410

Mariángel, S. V., \& Jiménez, J. E. (2015). Development of syntactic and phonological awareness in Chilean children: A cross-sectional study. Revista Latinoamericana de Psicologia, 48(1), 1-7. https://doi.org/10.1016/j.rlp.2015.09.010

Ministerio de Salud y Protección. (2012). RESOLUCION NUMERO 8430 DE 1993 (Octubre 4). Congreso de la República de Colombia., 32(4), 471-473. https://doi.org/10.7705/biomedica. v32i4.1526

Noguera, L. M., Herazo, Y., \& Vidarte, J. A. (2013). Correlation between psychomotor profile and logical mathematical performance in children 4 to 8 years. Revista Ciencias de la Salud, 11(2), 185-194.

Palacio-Duran, E., Pinillos-Patiño, Y., Herazo-Beltrán, Y., Galeano-Muñoz, L., \& Prieto-Suarez, E. (2017). Determinants of psychomotor performance in schoolchildren from Barranquilla, Colombia. Revista de Salud Publica, 19(3), 297-303. https://doi.org/10.15446/rsap. v19n3.65597

Pascual-Lacal, M., Madrid, D., \& Estrada-Vidal, L. (2018). Factores predominantes en el aprendizaje de la iniciación a la lectura. Revista Mexicana de Investigación Educativa; Mexico City, 23, 1121-1147.

Ramírez-Leyva, E. M. (2016). De la promoción de la lectura por placer a la formación integral de lectores. Investigacion Bibliotecologica, 30(69), 95-120. https://doi.org/10.1016/j. ibbai.2016.04.014

Rodriguez, G., \& Castillo, H. (2018). Tareas de búsqueda visual: Modelos, bases neurológicas, utilidad y prospectiva. Universitas Psychologica, 17(1), 1-12. https://doi.org/10.11144/ Javeriana.upsy17-1.tbvm

Rodríguez, M., \& Gaeta, M. (2018). Estrategias volitivas, orientación a metas y rendimiento académico en el nivel medio superior: un estudio exploratorio. Revista Digital A\&H, 86-97.

Salamanca, L. M., Naranjo Aristizábal, M. M. del C., \& González Marín, A. del P. (2013). validez y confiabilidad del cuestionario del trastorno del desarrollo de la coordinación versión en Español. Revista Ciencias de la Salud, 11(3), 263-274.

Sánchez-Panchuelo, R. M., Besle, J., Mougin, O., Gowland, P., Bowtell, R., Schluppeck, D., \& Francis, S. (2014). Regional structural differences across functionally parcellated Brodmann areas of human primary somatosensory cortex. NeuroImage, 93, 221-230. https://doi. org/10.1016/j.neuroimage.2013.03.044

Torres-Rivas, M., \& López-Gómez, S. (2017). La reeducación de las disgrafías: perspectivas neuropsicológica y psicolingüística. Pensamiento Psicológico, 15(1), 73-86. https://doi. org/10.11144/Javerianacali.PPSI15-1.RDPN 